\title{
Ultrawide Field of View by Curvilinear Projection Methods
}

\author{
Jonah Napieralla \\ Blekinge Institute of \\ Technology \\ Valhallav. 1 \\ SE-371 79 Karlskrona, \\ Sweden

\author{
Veronica Sundstedt \\ Blekinge Institute of \\ Technology \\ Valhallav. 1 \\ SE-371 79 Karlskrona, \\ Sweden
}

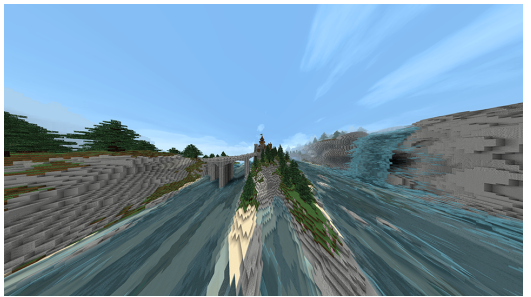

(a) Perspective

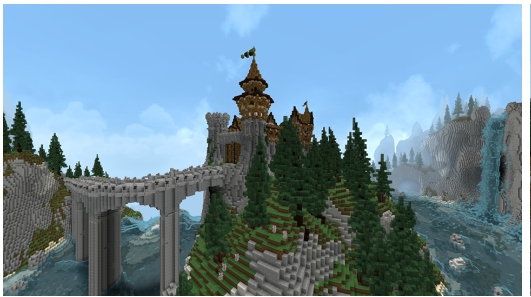

(b) Panini

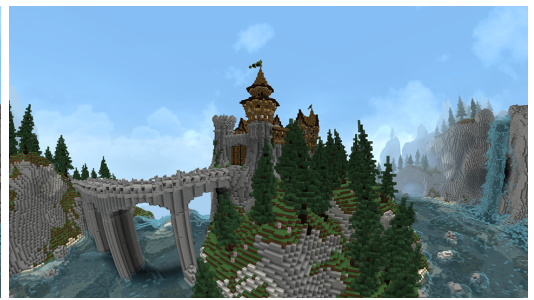

(c) Stereographic

Figure 1: One view, rendered at 170 degrees of field of view using different projection methods (a, b, c). Scenery provided by FALL Studios.

\begin{abstract}
The rectilinear Perspective projection produces natural-looking results on the condition that the degree of field of view (FoV) is narrow, as raising it causes an exponential increase in visual distortion. Curvilinear perspective projection methods that counter this issue exist in photography, but unlike the rectilinear Perspective projection, these are neither used nor technically documented in computer graphics. This paper contributes by presenting results from a perceptual experiment comparing the rectilinear Perspective projection method to the curvilinear Panini and Stereographic projection methods. These are commonly used with wide-angle lenses in photography and have been digitally recreated for use in computer graphics. The experiment shows a clear preference for the two curvilinear projection methods at high degrees of FoV, as not a single participant prefers the rectilinear Perspective projection at degrees of FoV approaching the human breadth of vision.
\end{abstract}

\section{Keywords}

Computer graphics, Perception, Human computer interaction (HCI), Graphical projection methods, Field of view, Perspective, Panini, Stereographic, Rectilinear, Curvilinear.

\section{INTRODUCTION}

The rectilinear Perspective projection method has always been the standard in realistic computer renditions. Of the projection methods made readily available by the common 3D graphics libraries, it is the only one that takes the user's positional perspective into account, a necessity for a natural look. But it does suffer from an unaddressed shortcoming; as the degree of FoV in-

Permission to make digital or hard copies of all or part of this work for personal or classroom use is granted without fee provided that copies are not made or distributed for profit or commercial advantage and that copies bear this notice and the full citation on the first page. To copy otherwise, or republish, to post on servers or to redistribute to lists, requires prior specific permission and/or a fee. creases, it experiences increasing amounts of distortion that leads to the rendition eventually becoming unrecognizable [Yan08], as demonstrated in Figure 2. The consequences of this have not yet been realized because the current hardware (computer monitors and TV screens) has not incentivized anything but unrealistically low degrees of FoV. But as ultrawide monitors and head-mounted VR-goggles attempt to push the limits of immersive digital experiences, the shortcomings of the Perspective projection method will have to be addressed.

Higher degrees of FoV have been shown to benefit the user in many ways, by, for example, allowing them to perform visual search tasks more effectively [Osm14], which makes them popular in gaming. In one of the most popular competitive 3D games at the time of writing - Overwatch - almost $99 \%$ of professional players 
use the highest degree of FoV available to them [Pro], a trend that spans many competitive games. As computer monitors have become wider, many applications have also started automatically setting the FoV higher for users of widescreen-monitors [Wgf], as monitors that cover a greater portion of the user's view are shown to merit the use of higher degrees of FoV [Ste11]. Assuming that the current trend continues; larger screens that encompass a greater portion of the user's view will become more prevalent in the future, and this increase in size will warrant increasingly higher degrees of FoV. However, that will only be feasible if the picture can be drawn without noticeable distortion. This issue merits the evaluation of alternative projection methods, and of whether they look natural enough to enable the use of high degrees of FoV.

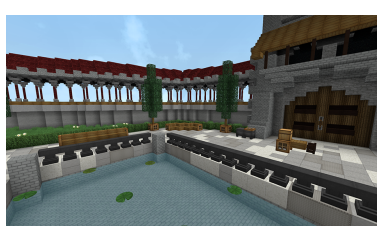

(a) 110 Degrees

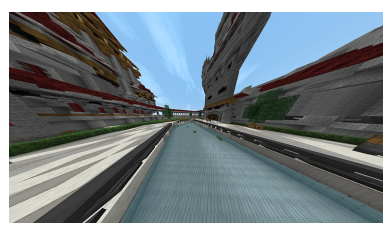

(c) 170 Degrees

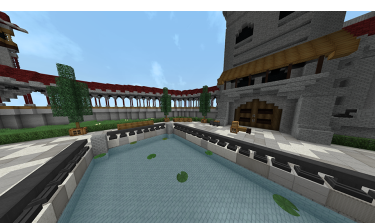

(b) 140 Degrees
Figure 2: Difference between 110, 140, and 170 degrees of FoV, using the Perspective projection method. Demonstrating the exponential increase in distortion.

Scenery provided by Elysium Fire.

\section{OVERVIEW OF PROJECTION METHODS}

In the process of converting the view of a virtual 3D world into a $2 \mathrm{D}$ rendition; projection methods are used to map points from the $3 \mathrm{D}$ world onto points on a 2D plane that can thereafter be drawn on a physical display. What differentiates projection methods is how they distribute these points onto the plane, as the resulting projection is produced. FoV is a parameter of these projection methods. It is defined by a numeric degree that specifies the angle from the left to the right extent of the view; the breadth of vision of the virtual camera.

The key difference between the Perspective projection and the two alternative projection methods evaluated, the Panini and the Stereographic projections, is that the Perspective projection is rectilinear while the Panini and the Stereographic projections are curvilinear, as visualized in Figure 3. What defines a rectilinear projection method is that it keeps the path between every pair of points in 3D space straight in the resulting projection onto the $2 \mathrm{D}$ plane, producing no unnatural curvature of straight lines. Curvilinear projection methods do not share this trait, as they bend lines that do not pass through the center of the projection. What differentiates the Panini and the Stereographic projection method is that the Panini projection only bends horizontal lines, whereas the Stereographic projection is fully curvilinear and will bend both horizontal and vertical lines. The Stereographic projection method also has the uncommon characteristic of being conformal, which implies that the angle at which curves intersect will be correctly preserved in the projection, despite other kinds of distortions.

The Panini projection method [Sha10] was included in this study because it had received very high ratings in a recent user study comparing projection methods using still pictures [Kim17]. The Stereographic projection method was included for its characteristics, that contrast strongly with the Perspective projection, making for an interesting comparison, and because it was commended as the best choice for general-purpose use in photography at high degrees of FoV, in a qualitative analysis of various projection methods [Fle95].

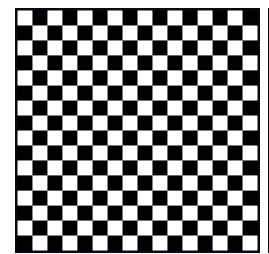

(a) Perspective

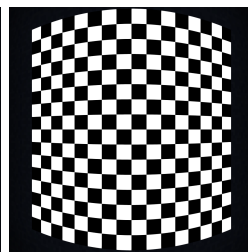

(b) Panini

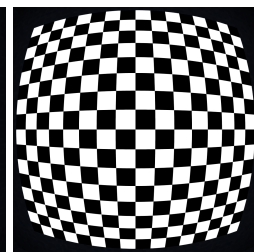

(c) Stereographic
Figure 3: Visualization of the point distribution and curvature of the three projection methods [Nap18].

\section{METHOD}

To assess the naturalness of the curvilinear Panini and Stereographic projections, and how they compare to the Perspective projection at high degrees of FoV, they were each rated in perceptual evaluations, by volunteering participants and then compared through a statistical analysis. The following sections present the data gathering process. For more information see [Nap18].

\subsection{Design}

The three projection methods were assessed in tests that simulated the most common usage scenarios of 3D software-applications, using a variety of virtual scenery that was intended to assess a wide enough spectrum of potential use. These tests were grouped into the following usage scenarios: 
- Photographic tests would present regular still pictures. These would allow the projection methods to be assessed more precisely, as it allowed the participants to carefully analyze the aesthetic qualities of each rendition.

- Cinematographic tests would consist of the camera moving, in both position and rotation, along a predefined path through a scene. Moving too fast for minute details to be discernible, these tests would instead show how points in space flow across the screen.

- Interactive tests would consist of the camera being fixed to a set vantage point, allowing only rotation, by the movement of the computer mouse. These would allow the participants to consider the feeling of actively engaging with an application employing the specific projection methods.

Because the photographic tests were expected to take the least time to rate, and the interactive tests the most time; four different sceneries were used in evaluating the photographic tests, three in the cinematographic, and two in the interactive. Each scenery would be employed in nine tests, as the Perspective, Panini, and Stereographic projection method each were evaluated at 110, 140, and 170 degrees of (horizontal) FoV, at a 16:9 aspect ratio. These degrees were chosen because it was expected that those below 110 would show too little distortion, and those above 170 too much distortion, to have produced valuable results.

\subsection{Rendering}

The experiment-application was built on top of a modified version of the game Minecraft [Moj], adjusted to fit the demands of the perceptual experiment. This approach was chosen because using a preexisting implementation of the projection methods would facilitate the development of the experiment, and an open-source rendering modification [Git], that had implemented the projection methods to be evaluated in this study, existed only for this application. To produce the curvilinear Panini and Stereographic projections, the rendering modification had to use an uncommon, and computationally less efficient approach, compared to rendering with the rectilinear Perspective projection. This involved first rendering five square views around the position of the camera, using the Perspective projection method; one in the direction that the camera was facing, as well as one 90 degrees to the left, to the right, above, and below that view, as shown in Figure 4. These views would all originate from the position of the camera, but they were rotated to be facing different directions. They were then sent to a fragment shader specific to the desired projection method, where they formed a theoretical cube around the origin that was ray-casted to for each pixel of the screen. Where the ray intersected with one of the view-planes, it sampled a color to output for that pixel. This process faithfully reproduced the Panini and the Stereographic projection from views rendered with the Perspective projection method, as the outcome would be virtually identical to ray-casting directly into the complex environment, but computationally a lot more efficient.

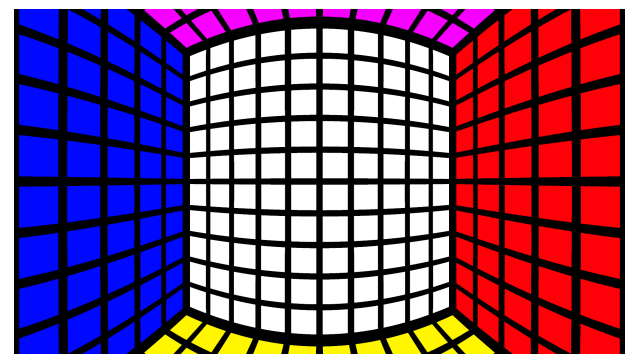

Figure 4: Picture taken from inside the cube that is raycast to produce the two curvilinear projection methods, shown using the Panini projection [Nap18].

\subsection{Perceptual Evaluation}

To assess each projection method at every degree of FoV; a perceptual experiment was conducted in which every participant rated a total of 81 tests, presented in randomized order. The average time to rate all tests took approximately 14.5 minutes. These were displayed on a 23-inch monitor, at an approximate distance of 71 centimeters from the participants' eyes, a distance that each participant was asked to maintain by keeping their head approximately aligned with a given point of reference. One test would be displayed at a time, consisting of a view that filled the entire screen, and it would be rated from within the experiment-application. Participants were asked the same question for each test; How unnatural (1) or natural (5) does the current projection look to you?, which was rated using a 5-point Likert scale. A total of 24 people took part, whose ages ranged from 18 to 29 years, and who used 3D software-applications at an average of approximately once a week.

\section{RESULTS}

Every projection method would generally be rated less favorably when the degree of FoV increased, as that would always increase distortion, but the Perspective projection method was affected more significantly by this increase than the Panini and Stereographic projections were. The drop in ratings can, for the most part, be considered exponential, as there was a lesser difference in ratings between 110 and 140 degrees than between 140 and 170 degrees. Only the Perspective projection would drop close to the lowest possible mean rating, 
as it fell from the universally highest ratings at 110 degrees to the lowest at 170 degrees, shown in Figure 5. While the Perspective projection method received relatively even mean ratings across the three usage scenarios, ratings of the Panini and Stereographic projections were less consistent. In the photographic and cinematographic tests, the Panini projection was rated only marginally lower than the Perspective projection at 110 degrees, with no significant difference between them ( $p$ $>0.05$ ) at that degree of FoV, but in the interactive tests, it dropped to unusually low ratings at every degree of FoV. The Stereographic projection received lower ratings than the Panini projection in the photographic tests, and in the cinematographic tests, its ratings fell further as those of the Panini projection rose. Only in the interactive tests would the Panini and Stereographic projections be rated similarly, with no significant difference between the two ( $p>0.05)$ at any degree of FoV. There was also no significant difference between the Perspective and Stereographic projections in the cinematographic tests at 140 degrees. Apart from the aforementioned results; there was a significant difference $(p$ $<0.05$ ) between ratings of every projection method employed in the same usage scenario and at the same degree of FoV. All statistical significance tests were computed using a Pairwise Wilcoxon signed-rank test.

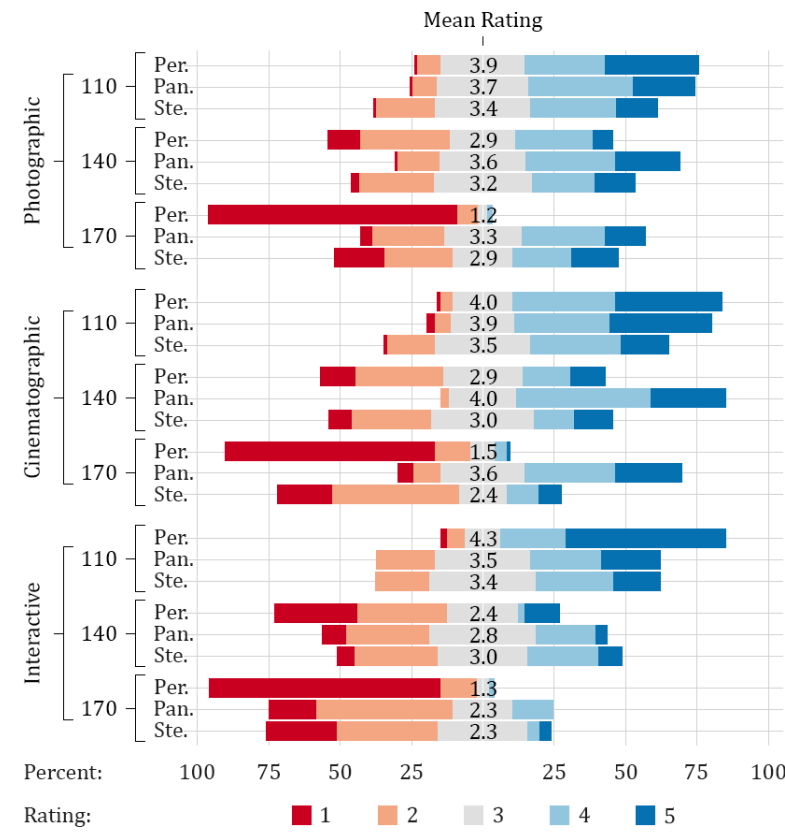

Figure 5: Distribution of ratings across tests visualized by their respective colors, for every projection method and at every degree of FoV, separated by scenario.

The mean change in individual participants' rating of a projection method, when only the scenery changed but not the projection method or FoV, was $(0.66,0.92,1.20)$ for the Perspective, Panini, and Stereographic projec- tion methods, respectively. Showing that the favorability of the curvilinear projection methods was more dependent on the scenery displayed. The distribution of individual participants' preferred projection method, across the three usage scenarios, calculated by counting every participant's ratings separately, was (63\%, $31 \%, 6 \%)$ at 110 degrees, $(19 \%, 69 \%, 12 \%)$ at 140 degrees, and $(0 \%, 90 \%, 10 \%)$ at 170 degrees, for the Perspective, Panini, and Stereographic projection methods, respectively. These differ from the mean ratings, as, for example, the Stereographic projection received higher overall ratings than the Perspective projection at 140 degrees, but was less often a preferred projection method.

\section{DISCUSSION}

Every usage scenario would be tested using a number of different sceneries, and ratings of the Panini and the Stereographic projection would vary significantly across these. The proximity of the foremost objects in the scenery appeared to be the pivotal aspect, as sceneries with objects in close proximity to the camera were rated unusually poorly. This is likely due to the curvilinear nature of these two projection methods, which arguably becomes much more noticeable when objects of distinct form take up a large portion of the view. When a distinct shape, such as a straight line, is distorted, the user will easily notice the abnormality as it deviates from their expectations of what something is supposed to look like, as the curved posts in Figure 6 (left). When objects lie close to the camera; shapes are likely to dominate the look of the scenery. Whereas if the foremost objects lie at a greater distance from the camera, then composition will dominate, and a composition of objects tends to be irregular, which renders any distortion less noticeable, as in Figure 6 (right).
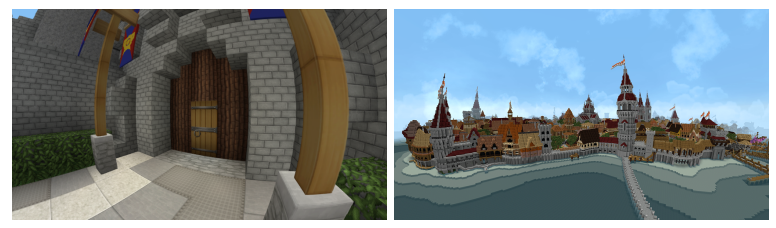

Figure 6: Difference between a focus on objects (left) and a focus on composition (right), demonstrated using the curvilinear Stereographic projection method at 170 degrees of FoV.

$$
\text { Scenery provided by Elysium Fire. }
$$

The further a point lies from the center of the screen, the more it is distorted. So when the display takes up only a small portion of the user's view, the distortion will remain very noticeable, as it lies physically close to the assumed center of focus, the center of the screen. However, when the display takes up a large portion of the user's view, then the distortion will lie physically 
further away from the center of focus, within the user's peripheral vision, where it might be less perceptible. Any unnatural curvature caused by either the Panini or the Stereographic projection method might then bother the user less. This theory would however not apply to the Perspective projection, as it suffers from a further problem; it distorts the proportions of the entire view. When the degree of FoV increases, parts of the view must shrink, as they must take up relatively less space on the screen to fit that which becomes visible with the additional degrees of FoV. However, the Perspective projection does not shrink the earlier view by a proportionally accurate amount. It shrinks it to a disproportionately small size, leaving the outer parts stretched beyond the proportions of the central parts which become inappropriately small, as illustrated in Figure 7.

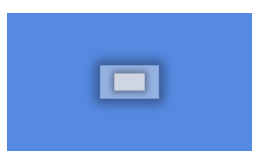

(a) Perspective

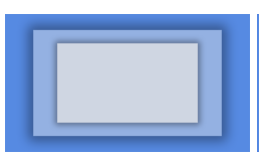

(b) Panini

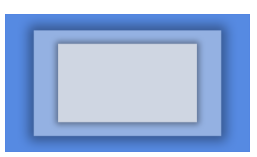

(c) Stereographic
Figure 7: Proportional differences between 110, 140, and 170 degrees of FoV, from inner to outer rectangle, illustrating a disparity between projection methods.

\section{CONCLUSIONS AND FUTURE WORK}

The Perspective, Panini, and Stereographic projection methods were evaluated in simulations of common usage scenarios, using different virtual scenery, by 24 volunteering participants of a perceptual experiment. The Perspective projection was shown to be the optimal choice at narrow degrees of FoV, as it was rated the most favorably in every test at 110 degrees, although often with an insignificantly small margin to the Panini projection. Because the Perspective projection was much more significantly affected by every increase in the degree of FoV, its ratings fell past the two curvilinear projection methods at 140 degrees. The Panini and the Stereographic projection were favored at 140 and 170 degrees, but their ratings depended largely on the usage scenario within which they were employed, as well as on the virtual scenery that was displayed. While the use of curvilinear projection methods has been justified, they will first become practically applicable when they can be computed efficiently enough not to inhibit the effective use of the applications they are employed in. Unless dedicated ray-tracing hardware becomes more prevalent in consumer devices, an investigation of alternative approaches to rendering with curvilinear projection methods, and the computing overhead associated with them, is merited. In addition, a further exploration of more realistic interactive experiences and spatial navigation tasks would be appropriate.

\section{REFERENCES}

[Fle95] Fleck, M. Perspective Projection: The Wrong Imaging Model. Department of Computer Science, University of Iowa, Technical Report TR 95-01, 1995.

[Kim17] Kim, Y.W. and Lee, C.-R. and Cho, D.-Y. and Kwon, Y.H. and Choi, H.-J. and Yoon, K.J. Automatic Content-aware Projection for 360 Videos. 2017 IEEE International Conference on Computer Vision (ICCV), pp.4753-4761, 2017.

[Moj] Mojang Synergies AB. Official Minecraft Website: https://minecraft.net. Online, accessed 27February-2020.

[Osm14] Malla Osman, Z. and Dupire, J. and Topol, A. and Cubaud, P. A Non Intrusive Method for Measuring Visual Attention Designed for the Study and Characterization of Users' Behavior in Serious Games. IARIA, International Journal On Advances in Internet Technology Vol. 7, pp.262271, 2014.

[Pro] ProSettings.net. Statistics on Video Settings of Professional Overwatch Players: https://www.prosettings.com/overwatch-prosettings. Online, accessed 27-February-2020.

[Sha10] Sharpless, T. K. and Postle, B. and German, D. M. Pannini: a new projection for rendering wide angle perspective images. Eurographics Association, Proceedings of the Sixth international conference on Computational Aesthetics in Graphics, Visualization and Imaging, pp. 9-16, 2010.

[Ste11] Steinicke, F. and Bruder, G. and Kuhl, S. Realistic Perspective Projections for Virtual Objects and Environments. ACM Trans. Graph., pp.112:1112:10, 2011.

[Wgf] Widescreen Gaming Forum. Widescreen Gaming Forum article about Hor+ Technique: http://www.wsgf.org/article/screen-change. Online, accessed 27-February-2020.

[Yan08] Yankova, A. and Franke, I. Angle of View vs. Perspective Distortion: A Psychological Evaluation of Perspective Projection for Achieving Perceptual Realism in Computer Graphics. ACM, Proceedings of the 5th Symposium on Applied Perception in Graphics and Visualization, pp. 204-204, 2008.

[Git] 18107. Render 360 Mod GitHub Repository: https://github.com/18107/MC-Render360. Online, accessed 27-February-2020.

[Nap18] Napieralla, J. Comparing Graphical Projection Methods at High Degrees of Field of View. Blekinge Institute of Technology, Bachelor Thesis, 2018. 